\title{
Theoretical High Pressure Study of Phonon Density of State and Debye Temperature of Solid $\mathrm{C}_{60}$ : Grüneisen Approximation Approach
}

\author{
Adnan M. Al Sheikh*1, Sirwan K. Jalal ${ }^{2}$, Salar A. Mawlood ${ }^{3}$ \\ ${ }^{1}$ Department of Physics, University of Mosul, Iraq \\ ${ }^{2}$ Department of General Science, Charmo University, Iraq \\ ${ }^{3}$ Department of Physics - Salahaddin University, Iraq \\ E-mail: 1dr.adnan@uomosul.edu.iq
}

Received 1 July 2021, Revised 27 September 2021, Accepted 2 November 2021

\begin{abstract}
A nanomaterial equation of state has been combined with Grüneisen approximation in present work to investigate the influence of high pressure on phonon density of state function of $\mathrm{C}_{60}$ through evaluating variations of lattice vibration frequencies and variation of mode density. Furthermore, the effect of high pressure on Debye temperature has been determined by using a formula of volume dependence of Debye temperature. Volume compression ratio in $\mathrm{C}_{60}$ has been computed, using a nanomaterial equation of state. Expressions of pressure dependence of phonon density of state and Debye temperature have been combined with volume compression ratio value. The evaluated result of volume compression ratio and phonon density of state have been compared with the experimental observations and good agreement can be seen.
\end{abstract}

Keywords: $C_{60}$, high pressure; Grüneisen approximation; phonon density of state; Debye temperature.

\section{Introduction}

A new structural form of solid carbon is $\mathrm{C}_{60}$. Carbon nanotubes as integrate future of $\mathrm{C}_{60}$ have potential applications in the engineering industry and medical areas due to its unusual elastic properties. $\mathrm{C}_{60}$ has got intensive attention by researchers to describe its advantageous properties. The external mode spectrum of solid carbon under hydrostatic pressure up to $9 \mathrm{Gpa}$ using elastic neutron scattering has been investigated [1].

An X-ray diffraction experiment has been established on $\mathrm{C}_{60}$ using diamond anvil cell and up to pressure 28(Gpa) [2]. The pressure dependence lattice parameter and volume compression ratio were observed. A tight-binding molecular dynamics method used, for investigating phonon dispersions and density of states for solid $\mathrm{C}_{60}$ [3]. Equation of state of $\mathrm{C}_{60}$ has been found experimentally up to pressure of about $10 \mathrm{Gpa}$ [4], and theoretically using different equations of state $[5,6]$. Volume compression ratio, bulk modulus and spinodal pressure of $\mathrm{C}_{60}$ have been calculated by using various EOSs [7]. Moreover, in an intensive study, the validity of some equations of state of carbon nanotubes investigated [8].

In order to find solutions to a variety of problems in condensed phase of physics, equations of state that accurately predict solids behavior at high pressure and temperature are required. The study of $\mathrm{C}_{60}$ with varying pressure can help us to study many crystal forms of solid materials. This work is an attempt for investigating the effect of pressure on phonon density of state of $\mathrm{C}_{60}$ and Debye temperature, using a nanomaterial equation of state (EOS) [9]. In present work, pressure corresponding to volume compression ratio was evaluated by means of the EOS.

Debye temperature is an important quantity for determining various properties of solids at high pressure and high temperature. Numerous efforts have been taken for describing Debye temperature of different materials at high pressure condition [10].

The values of Grüneisen parameter and Debye temperature at ambient condition, used in the present study were calculated in literature [11]. Three body force shell model have been used to evaluate Debye temperature and Grüneisen parameter of $\mathrm{C}_{60}$ at atmospheric pressure and room temperature [11].

As Grüneisen parameter describes the effect of changing volume of solid phase on vibrational motions of atoms, therefore, the effect of decreasing volume of the lattice by applying high pressure, will result in changing the phonon density of state.

\section{Theoretical analysis \\ 2.1 Equations of state}

The relationship between thermo dynamical variables; pressure $(\mathrm{P})$, volume $(\mathrm{V})$ and temperature $(\mathrm{T})$ known as equation of state, with the help of EOSs, one can describe various properties of solid under varying conditions of pressures or temperatures. Based on different assumptions, many equations of state have been derived in literature. The equations of state are classified in to Thermal- pressure equation of state (Th-P EOS) and isothermal EOS.

\section{Thermal- pressure (Th-P EOS):}

At a given volume $\mathrm{V}$ and temperature $\mathrm{T}$, the pressure $\mathrm{P}$ is descried as by the summation of two parameters:

$$
P(V, T)=P_{\text {initial }}\left(V, T_{0}\right)+\Delta P_{t h}(V, T)
$$


Where $P_{\text {initial }}$ represents pressure required for compressing the sample material from initial volume $\left(\mathrm{V}_{0}\right)$ at ambient state $\left(\mathrm{P}=0\right.$ and $\left.\mathrm{T}_{0}\right)$ to another volume $\mathrm{V}$ at constant temperature $\mathrm{T}_{0}$.

$\Delta \mathrm{P}_{\text {th }}$ is the change in thermal pressure.

Thermal pressure arises due to the variation in temperature from $\mathrm{T}_{0}$ to $\mathrm{T}$ at a constant volume.

If the final volume $\mathrm{V}=\mathrm{V}_{0}$ at pressure $\mathrm{P}$ and temperature $\mathrm{T}$ at the initial conditions, therefore, to obtain this final volume $\mathrm{V}$ no pressure is required i. e $\mathrm{P}_{\text {initial }}=0$, in this case $\mathrm{P}\left(\mathrm{V}, \mathrm{T}_{0}\right)=\Delta \mathrm{P}_{\text {th }}$ as illustrated in Fig.1 (black line).

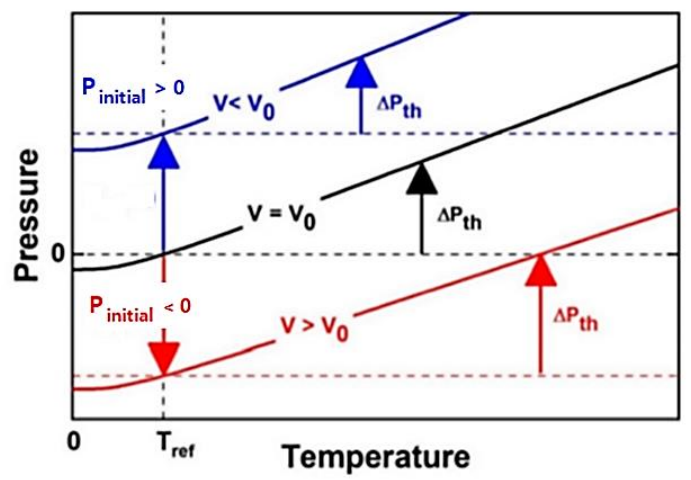

Figure 1. The description of different pressures and variables used in Th-P EOS, showing three various volumes [12].

Compression at the initial state is necessary to reach the final volume $\mathrm{V}$, so that $\mathrm{P}_{\text {initial }}=0$. In this case, the total pressure is $\Delta \mathrm{P}_{\text {th }}$ (black line) in the above figure. Under a positive pressure $\mathrm{P}_{\text {initial }}$ above isochoric pressure the volume $\mathrm{V}$ is smaller than $\mathrm{V}_{0}$. On the other hand, for pressure $\mathrm{P}$ and temperature $\mathrm{T}$ conditions under the isochoric pressure the $\mathrm{V}$ $>\mathrm{V}_{0}$, the material expands at $\mathrm{T}_{0}$ to reach the required $\mathrm{V}$. This pressure is called spinodal pressure where $\mathrm{P}_{\text {initial }}<0$ (red in Fig. 1).

\section{Mie-Grüneisen-Debye (MGD) EOS:}

Thermal-pressure in solid crystal generate from the excitation of its vibrational modes. Thermal-Pressure EOS depends on several assumptions. Therefore, calculating the thermal-pressure and hence its variation $\left(\Delta \mathrm{P}_{\text {th }}\right)$ from the initial temperature is used to distinguish diverse thermalpressure EOSs. The statistical mechanics of the vibrational motion of atoms (phonons) of crystalline solids as a quasiharmonic approximation QHA, leads to the derivation of MGD EOS.

QHA assumes that the atoms of the material are vibrating independently, without any interaction, and that their wave vectors are only dependent upon the molar volume.

phonon-mode Grüneisen parameter describes the phonon frequency dependent of the volume of the crystalline solids.

$$
\gamma_{i}=\frac{V}{\omega_{i}} \frac{\partial \omega}{\partial V}
$$

To represent a single Grüneisen parameter $\gamma$,we assume that all of the phonon frequencies have the same volume dependence. Now, the Grüneisen parameter $\gamma$ is assumed to be volume dependent by the MGD $\operatorname{EOS} \gamma=\gamma_{0}\left(\frac{V_{P}}{V_{0}}\right)^{q}$, where, $\mathrm{q}$ is the second Grüneisen parameter, Thus two
Grüneisen parameter are associated to the vibrational frequencies, $\mathrm{q}$ is larger than zero and its influence is to increase the value of first Grüneisen parameter $\gamma$ at high temperatures. Moreover, the value of second Grüneisen parameter $\mathrm{q}$ determines how fast the value of $\gamma$ is changed, especially at large volumes.

In quantum-statistical framework, the vibrationrotational and intramolecular contributions of crystalline molecules, as isotropic quantum oscillators are associated with two Grüneisen parameters, i.e. $\gamma$ and $q[13]$.

The thermal pressure becomes $P_{t h}=\frac{\gamma}{V} E_{t h}, V$ indicates molar volume, and $E_{\text {th }}$ is the energy of the vibrational modes which indicated the thermal energy.

$E_{\text {th }}$ is derived from a phonon density of states, which can be represented by a Debye temperature $\theta_{\mathrm{D}}$. Further assumption $E_{\text {th }}$ is represented by the Debye function [14]. Therefore, if a finite temperature is considered for $\mathrm{T}_{0}$, the change in $P_{\text {th }}$ from $\mathrm{T}_{0}$ becomes:

$$
\Delta P_{\text {th }}=-\frac{3 \mathrm{nR} \gamma}{V}\left[T \times D\left[\frac{\theta_{D}}{T}\right]-T_{r e f} \times D\left[\frac{\theta_{D}}{T_{0}}\right]\right]
$$

$D\left[\frac{\theta_{D}}{T}\right]$, is the Debye function, $\mathrm{R}$ represents the gas constant, $\mathrm{n}$ is the number of atoms and $\mathrm{T}_{\text {ref }}$ is the reference temperature.

\section{Isothermal EOS:}

The Pressure dependence of the volume of a material at constant temperature $T_{0}$ is characterized by isothermal equation of state. Based on different assumptions, various isothermal EOSs have been developed. In this study, an isothermal EOS is derived for Nano-size materials in terms of pressure $\mathrm{P}$, as expressed in the following [9]:

$$
P=B_{0}(1-\eta)+\frac{1}{2} B_{0}\left(B_{0}^{\prime}+1\right)(1-\eta)^{2}
$$

where,

$\mathrm{P}$ is the pressure

$\eta=\frac{V_{p}}{V_{0}}=$ volume compression ratio

$V_{0}$ and $\mathrm{V}_{\mathrm{p}}$ are volume at ambient and high pressure respectively.

$\mathrm{B}_{0}$ : Bulk modulus at atmospheric pressure.

$\mathrm{B}_{0}$ : First pressure derivatives of bulk modulus.

\subsection{Phonon Frequency Spectrum (PFS)}

Atoms of crystalline solid phase vibrate around their equilibrium position in the same manner as harmonic oscillators. Thus, the vibrational motion of all atoms represents an elastic wave which is quantized and named phonons. According to Debye approximation, the vibration of each atom corresponds to a normal mode (phonon). Thus, there are a wide range of vibrational modes present in the crystal. The number of vibrational modes in the range of frequency $\omega$ to $\omega+d \omega$ is termed as density of state $D(\omega)$ or phonon frequency spectrum. One of the most outstanding investigations relating to $\mathrm{C}_{60}$ is its phonon frequency spectrum which has been taken considerable in this study. The phonon density of state of $\mathrm{C}_{60}$ at atmospheric pressure 
was found by [15] and plotted in Fig. 3, as will be shown in section 3.2 in present work.

\subsection{Pressure dependence of vibrational modes}

From the definition of Grüneisen parameter, phonon frequencies $\omega_{\mathrm{i}}$ are volume dependent and, that is described by the mode Grüneisen parameters $[16,17]$.

$$
\gamma_{i}=-\frac{\partial \ln \omega_{i}}{\partial \ln V}
$$

The effect of pressure on the vibrational spectrum of a crystalline solid at constant temperature is described by the isothermal mode Grüneisen parameter, by the following relations. The isothermal bulk modulus $\mathrm{B}_{T}$ is

$$
\begin{gathered}
B_{\mathrm{T}}=-V \frac{\partial P}{\partial \mathrm{V}} \rightarrow \frac{\partial \mathrm{V}}{\mathrm{V}}=-\frac{\partial P}{B_{\mathrm{T}}} \\
\partial \ln \omega_{\mathrm{i}}=\frac{\partial \omega_{\mathrm{i}}}{\omega_{\mathrm{i}}} \\
\partial \ln V=\frac{\partial V}{V}=-\frac{\partial P}{B_{T}} \\
\partial \ln V=-\frac{\partial P}{B_{T}}
\end{gathered}
$$

Substituting eq. (6c) and eq. (6b) into eq. (5), it becomes to

$$
\begin{gathered}
\gamma_{i}=\frac{-\left(\partial \omega_{i} / \omega_{i}\right)}{\left(-\partial P / B_{T}\right)} \\
\gamma_{i}=\frac{B_{T}}{\omega_{\mathrm{i}}}\left(\frac{\partial \omega_{i}}{\partial P}\right)_{T}
\end{gathered}
$$

The theory predicts a linear dependence of vibrational frequency on pressure, but is only expected to be applicable for pressures that are small compared to the bulk modulus. The effect of pressure on lattice frequency and mode density of state as is [18].

$$
\omega_{\mathrm{P}}=\omega\left(\frac{V_{P}}{V_{0}}\right)^{-\gamma}
$$

$D_{P}\left(\omega_{P}, V_{P}\right)=D\left(\omega, V_{0}\right)\left(\frac{V_{P}}{V_{0}}\right)^{\gamma}$

where

$\omega_{P}$, is the lattice frequency at pressure $\mathrm{P}$

$\omega$, Lattice vibration at atmospheric pressure

$D_{p}\left(\omega_{P}, V_{P}\right)$ and $D\left(\omega, V_{\mathrm{o}}\right)$ are density of state at pressure $\mathrm{P}$ and atmospheric pressure respectively.

\subsection{Debye temperature $\left(\theta_{\mathrm{D}}\right)$}

Debye temperature $\left(\theta_{D}\right)$ or Debye characteristic temperature is the characteristic of each substance appearing in Debye theory of specific heats and given by:

$$
\theta_{D}=\frac{\hbar \omega_{D}}{k_{B}}
$$

Where $\theta_{D}$ - is the Debye temperature at atmospheric pressure $k_{B}$-Boltzmann constant

$\omega_{D}$ - Debye frequency at atmospheric pressure.

In the Debye model a solid is consider as an elastic continuum, which has a band of frequencies and the total number of vibrational modes is equal to the total number of degrees of freedom. As vibrational frequencies depend on equilibrium position which is change with pressure as a result of $\mathrm{V}_{\mathrm{p}} / \mathrm{V}_{0}$ variation with the pressure. Pressure dependence of Debye temperature is expressed as [ 8,19]:

$$
\theta_{P}=\theta_{D}\left(\frac{V_{P}}{V_{0}}\right)^{-\gamma}
$$

Where $\theta_{P}$ : Debye temperature under high pressure.

\section{Calculation and Results}

3.1. Evaluation of $V_{p} / V_{0}$ of $\mathbf{C}_{\mathbf{6}}$

Table 1. Values of the input parameters of $C_{60}$, at ambient pressure and room temperature.

\begin{tabular}{ll}
\hline Parameters & Value \\
\hline Bo & $18.1 \mathrm{Gpa}[4]$ \\
B $_{0}{ }^{\prime}$ & $15.7[4]$ \\
$\gamma$ & $2.899[11]$ \\
$\theta_{\mathrm{D}}$ & $182.76 \mathrm{~K}[11]$ \\
\hline
\end{tabular}

Table 2: Calculated Values of $V_{p} / V_{0}$ versus pressure

\begin{tabular}{cc}
\hline Pressure (Gpa) & $\mathrm{V}_{\mathrm{p}} / \mathrm{V}_{0}$ \\
\hline 0 & 1 \\
0.5976 & 0.97 \\
1.3043 & 0.94 \\
2.4163 & 0.90 \\
3.3777 & 0.87 \\
4.4483 & 0.84 \\
6.0454 & 0.80 \\
7.3706 & 0.77 \\
\hline
\end{tabular}

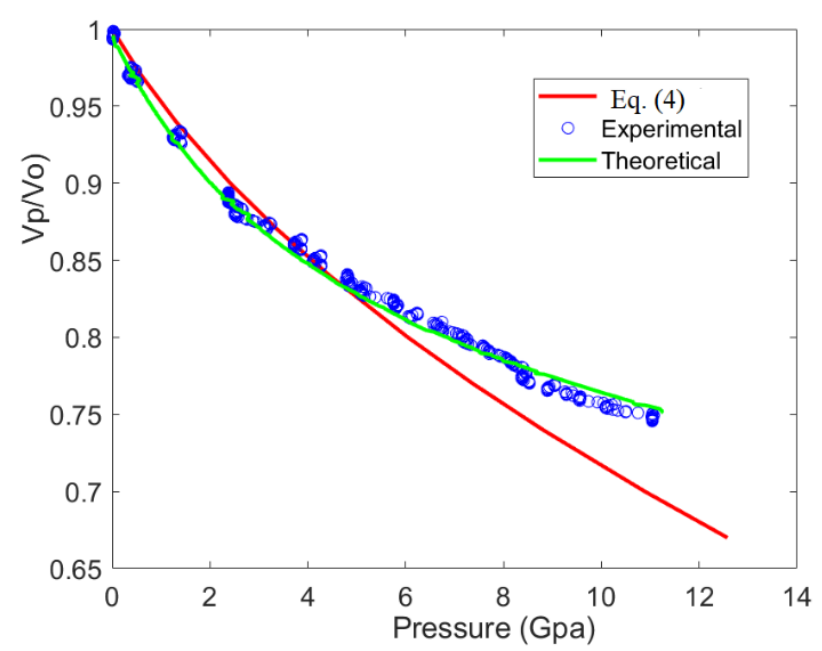

Figure 2. Variation of $V_{P} / V_{O}$ under high pressure by using the EOS, Eq.(4), experimental data [4]and theoretical data from [5]. 
On substituting $\mathrm{B}_{0}$ and $\mathrm{B}_{0}{ }^{\prime}$ values for $\mathrm{C}_{60}$ from Table 1 into equation 1 , and choosing $\mathrm{V}_{\mathrm{p}} / \mathrm{V}_{0}$ from 1 descending to 0.77. Variation of $\mathrm{V}_{\mathrm{p}} / \mathrm{V}_{0}$ with high pressure for $\mathrm{C}_{60}$, has been evaluated and the results are tabulated in Table 2, and shown in Fig .2. For comparison purpose, relevant experimental and theoretical data for volume compression ratio are displaced in fig.2. It is seen that our considered EOS fits the both literature results up to pressure range of $6 \mathrm{Gpa}$. Then, beyond this limit, the curve expects a more dramatic decrease rather than experimental and theoretical data.

\subsection{Evaluation of $\mathrm{C}_{60}$ Phonon Frequency Spectrum (PFS) under high pressure}

The PFS of $\mathrm{C}_{60}$ at room temperature and atmospheric pressure is illustrated in Fig.3 [15]. Where density of state is measured in arbitrary unit.

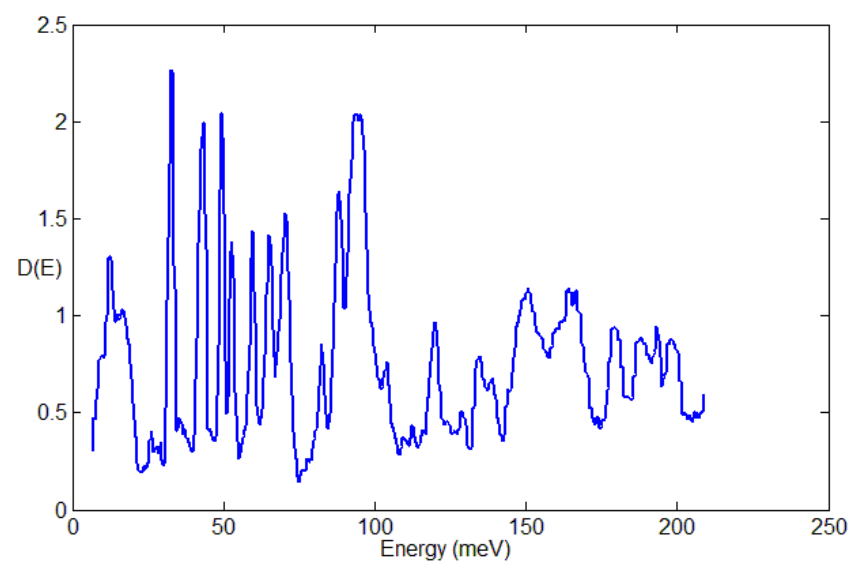

Figure 3. PFS of $C_{60}$ at atmospheric pressure and room temperature [15].
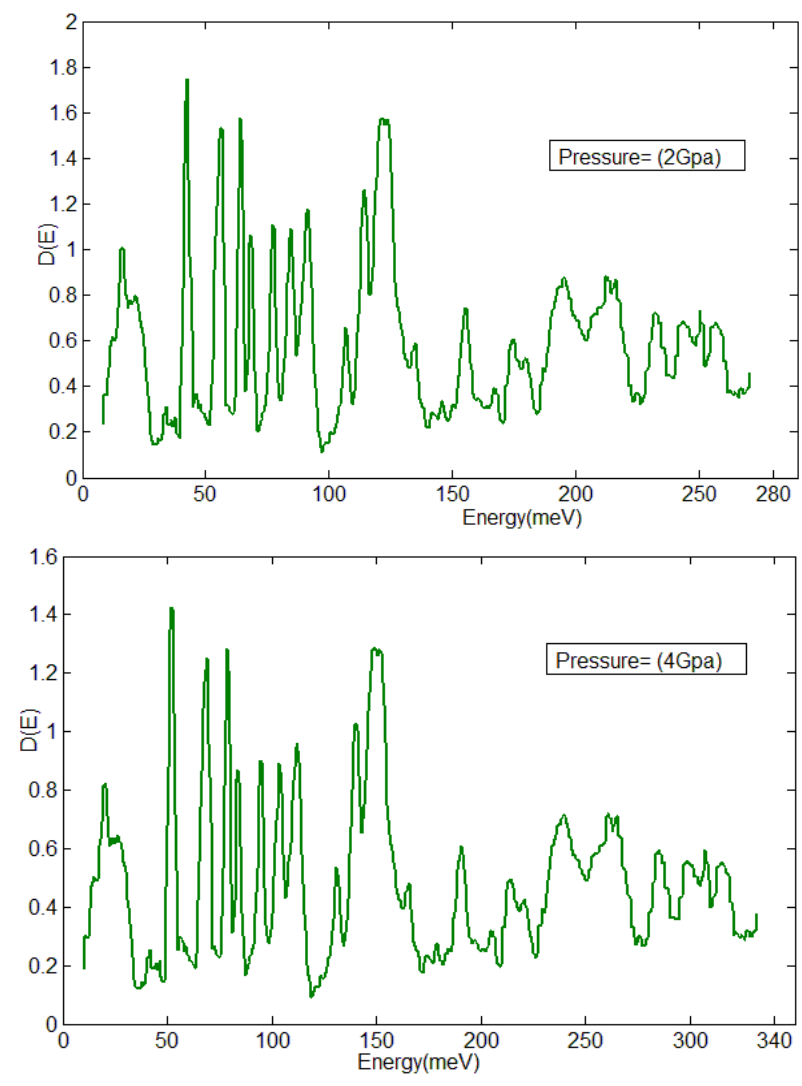

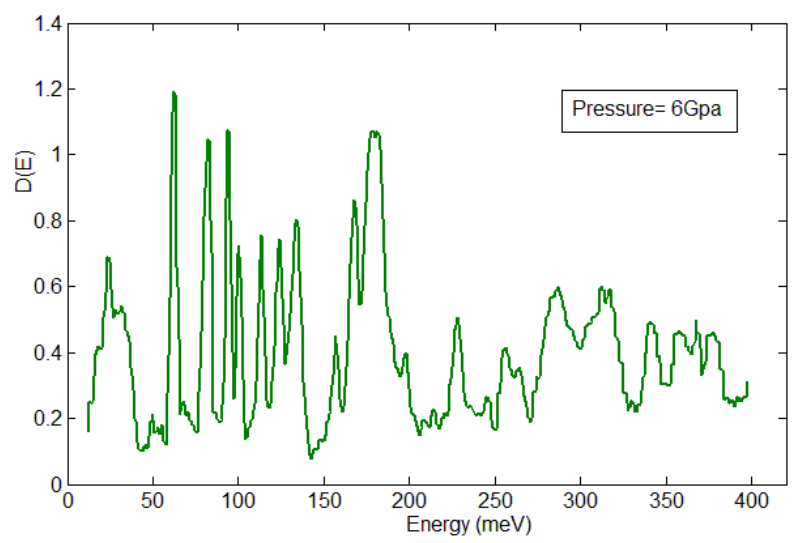

Figure 4. PFS of $C_{60}$ at various pressure ranges and room temperature.

Equations (9 and 10), represent the pressure dependence of phonon energy (frequency) and density of state. Combining eq. 4 with eqs. 9 and 10, phonon frequency spectrum of $\mathrm{C}_{60}$ has been computed under different high pressure values and the results are shown in Figs.4.

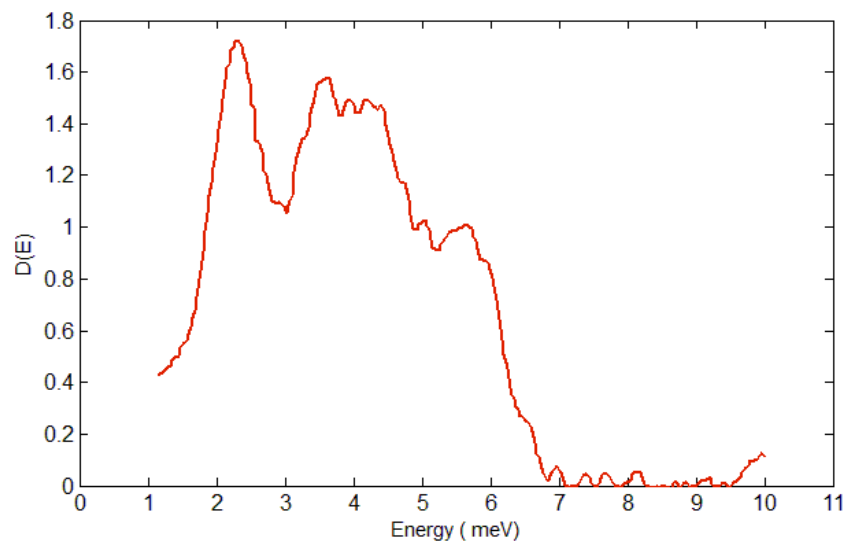

Figure 5. Experimantal PFS at ambient presssure and $180 K[1]$.

In order to support our results, Fig. 5 represents experimental PFS at ambient pressure and 180K [1] obtained by inelastic neutron scattering.

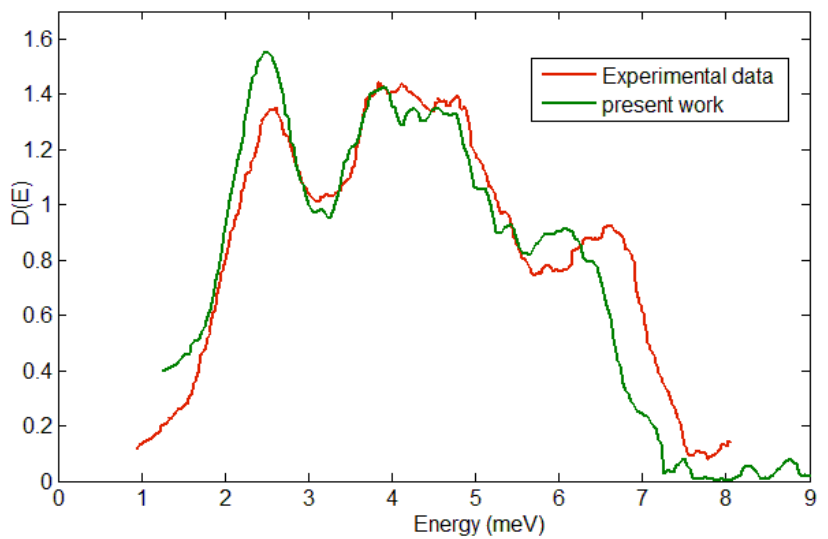

Figure 6. Experimental PFS of the external mode at $P=0.5 G p a$ and $T=290 K[1]$, in comparison with the same spectrum evaluated in the present work.

Neglecting temperature effect and using Grüneisen approximation given in eqs.(6 and 7), Fig. 6 shows experimental data[1] for pressure dependence of the external mode spectrum of $\mathrm{C}_{60}$ as a function pressure at $0.5 \mathrm{GPa}$ and 
$290 \mathrm{~K}$ obtained by using inelastic neutron scattering in comparison with the pressure dependence of the same spectrum, evaluated theoretically in present work by applying Grünesein approximation. It is observed that a very good agreement has been achieved as the effect of temperature on the spectrum is negligible.

\subsection{Evaluation of Debye Temperature $\left(\theta_{P}\right)$ of $\mathrm{C}_{60}$ under high pressure}

Choosing value of $\gamma$ and $\theta_{\mathrm{D}}$ from Table1. Then, substituting $\mathrm{V}_{\mathrm{p}} / \mathrm{V}_{\mathrm{o}}$ values from Table 2 into eq. 12. Debye temperature under high pressure $\left(\theta_{P}\right)$ has been calculated and the result shown in Fig.7. It is seen that the $\theta_{\mathrm{D}}$ variation rises linearly as the pressure increases.

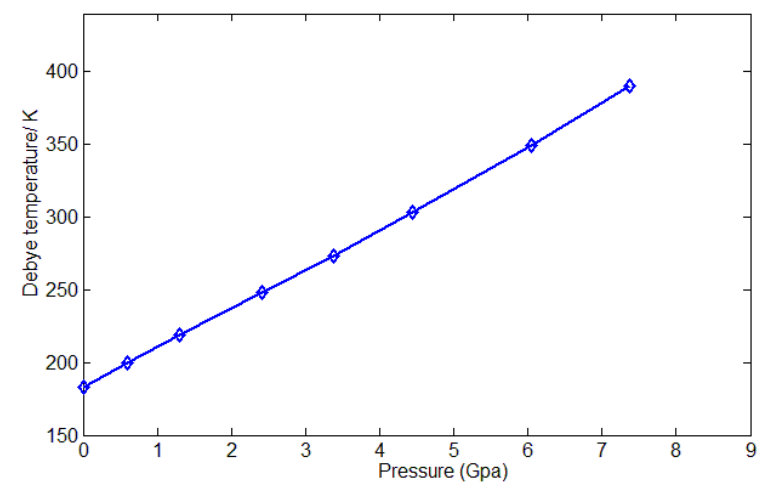

Fig.7. Pressure dependence of Debye temperature $\left(\theta_{P}\right)$ of $C_{60}$ using the EOS.

\section{Discussion}

The results of density of states $\mathrm{D}(\mathrm{E})$ and phonon energy under three different values of pressure are shown in Fig. 4. It is observed from Figs.4 that the phonon energy shifted towards higher energies with increasing pressure, while the density of states $\mathrm{D}(\mathrm{E})$ has dropped down, thus under high pressure more inactive modes become oscillating. This in line with theoretical predictions [20] that the frequencies of phonon frequency spectrum of solid materials shift towards higher frequencies, and some inactive modes become active. These results can be interpreted that as the pressure applied to a crystalline solid, lattice spacing parameter gets decreased, thus the wave number space is expanded.

The experimental results in Fig.6 supports what was obtained in Figs. 4 by comparing the results obtained by applying Grüneisen approximation on spectrum of Fig.5 with experimental spectrum under high pressure [1]. For, as well as a good agreement between both experimental data and present theoretical in Fig.6. Both show frequencies shift towards higher energies and the mode density decreases and becomes wider.

Further interesting study, is the computation of Debye temperature of $\mathrm{C}_{60}$ under strong compression. The pressure corresponding to $\mathrm{V}_{\mathrm{p}} / \mathrm{V}_{0}$ values calculated by the EOS given in eq. 4 and reported in Table 1. Equation (12) has been used to compute Debye temperature $\theta_{\mathrm{D}}$ under high pressure. The variation of Debye temperature at high pressure is shown in Fig. (7). It illustrates that Debye temperature increases linearly with pressure, since $\theta_{D}$ is directly proportional with $\omega_{D}$ and equation (8) implies that vibrational frequencies increase with pressure as well.

\section{Conclusion}

The present study analyzes the variety forms of EOSs that formulated on the basis of special assumptions and conditions. First, thermal- pressure EOS and its limitation is characterized. Second, as long as isothermal EOSs are identified, a nanomaterial isothermal EOS is used to perform a statistical understanding of effects of high pressure $\mathrm{P}$ on the volume V, Debye temperature and phonon frequency spectrum of Nano $\mathrm{C}_{60}$. The combinations of the results of volume compression ratio attained with the nanomaterial EOS in fig. 2 with equations $(9,10$ and 12) results in figures show the novelty of the entire calculation, as it agrees well with the experimental data shown in fig.6.

\section{Nomenclature}

$\begin{array}{ll}\mathrm{C}_{60} & \text { Fullerene carbon } \\ \mathrm{GPa} & \text { Gaga Pascal } \\ \mathrm{K} & \text { Kelvin } \\ \eta & \text { eta } \\ E_{\mathrm{th}} & \text { thermal energy } \\ \mathrm{V} & \text { volume } \\ \mathrm{P} & \text { pressure } \\ \mathrm{V}_{\mathrm{P}} / \mathrm{V}_{0} & \text { volume compression ration } \\ \mathrm{V}_{\mathrm{P}} & \text { volume under high pressure } \\ \mathrm{V}_{0} & \text { volume at ambient condition } \\ \gamma & \text { Grüneisen parameter } \\ \mathrm{q} & \text { second Grüneisen parameter } \\ \theta_{D} & \text { Debye temperature } \\ \theta_{\mathrm{P}} & \text { Debye temperature under high pressure }\end{array}$

\section{References:}

[1] H. Schober, B. Renker, "Pressure dependence of the external mode spectrum of solid $\mathrm{C}_{60}$," physical review B 59 (5), 3287,1999.

[2] J. Haines, J. M. Leger, “An X-ray diffraction study 0f $\mathrm{C}_{60}$ up to 28GPa," Solid State Communications, 90 (6), 361,1994 .

[3] J. Yu, R. K. Kalia, P. Vashishta, "Phonon dispersion and density of states of solid $\mathrm{C}_{60}$, " Applied Physics Letters 63(93), $3152,1993$.

[4] T. Horikawa, T. Kinoshita, K. Suito, and A. Onodera, Solid State Commun. 114, 121 (2000).

[5] S. Sh. Rekhviashvili, "Equation of State for Fullerite C60" Physics of the Solid State, 2017, Vol. 59, No. 4, pp. 835-837.

[6] S. Kareem Jalal, S. Ali mawlood, "Size dependent thermodynamic properties of nanoparticles, "International Journal of Thermodynamics, Vol. 23 (No. 4), 245-250,2020.

[7] A. M. Al-sheikh, S. K. Jalal, R. H. Al-saqa, "Equation of State and Thermo Dynamic Behaviour of $\mathrm{C}_{60}$ under High Pressure," Universal Journal of Mechanical Engineering 8(1), 59,2020.

[8] R. S. Preston, S. S. Hanna, J. Helerle, " Mössbaruer effect in metallic Iron. Phys. Rev., "128( 5) 22071962.

[9] D. Sharma, M. Kumar, "Effect of pressure on nanomaterials," Physica B, 405(13), 2820,2010. 
[10] S. K. Sharma, "Debye temperature of hep Iron at extreme compression, Solid State Communications, " 149 (47), 2207,2009.

[11] P. Singh, N. K. Gaur, "Thermal and elastic properties of $\mathrm{C}_{60}$ in FCC phase, " Sop transactions on theoretical physics, 1(2) 68 ,2014

[12] R. J. Angel, F. Miozzi, M. Alvaro," Limits to the Validity of Thermal-Pressure Equations of State" Minerals 9, 562,2019.

[13] S. S. Rekhviashvili, "Equation of state for Fullerite $\mathrm{C}_{60}$, Physics of the Solid State, " 59(4), 835, 2017.

[14] Anderson, O.L. Equations of State of Solids for Geophysics and Ceramic Science; Oxford University Press: Oxford, UK, 1995; p. 432.

[15] J. Yu, R. K. Kalia, P. Vashishta, "Phonon dispersion and density of states of solid $\mathrm{C}_{60}$," Applied Physics Letters 63(93), $3152,1993$.
[16] L. Burakovsky, D. L. Preston, "An analytical model of Grüneisen parameter at all densities," Journal of physics and chemistry of solids, 652002.

[17] S.Kareem Jalal, A. M. Al-Sheikh, R. H. Al-Saqa, “ High Pressure Effects on the Phonon Frequency Spectrum of Silicon Nanoparticle," Iran J Sci Technol Trans Sci 45, 391-396, 2021.

[18] J. L. Dlouha, "The influence of pressure on the Mössbauer effect, " Czech. J. Phys. B 14 (8), 57, 1964.

[19] S. Kareem Jalal, A. M Al-Sheikh, “ Theoretical high pressure study for thermoelastic properties of $\mathrm{NaCl}$ B1m” Rafidain J Sci 25:80-89, 2014.

[20] P. M. Sherwood, "Vibrational spectroscopy of solids, " Cambridge university press, 182(1972). 